\title{
Thermomorphogenic and Photoperiodic Responses of Nephrolepis exaltata 'Dallas Jewel'
}

\author{
John E. Erwin \\ Department of Horticultural Sciences, University of Minnesota, St. Paul, \\ MN 55108 \\ Royal D. Heins and James E. Faust \\ Department of Horticulture, Michigan State University, East Lansing, \\ MI 44824
}

Additional index words. fern, development rate, temperature, difference

\begin{abstract}
Nephrolepis exaltata (L.) Schott 'Dallas Jewel' plants were grown for 92 days under 16 day/night temperature (DT/NT) regimes and two photoperiods for a total of 32 environments. Temperatures ranged from 15 to $30 \pm 1.5 \mathrm{C}$. Photoperiod was either 9 hours (short days) or 9 hours plus a 4-hour night interruption (long days) using incandescent lamps. Photoperiod had no significant effect on either morphology or development rate. Frond length and leaflet count per frond were highly correlated with the average daily temperature (ADT). Frond length increased from 9.3 to $21.9 \mathrm{~cm}$ and leaflet count increased from 21 to 42 leaflets per frond as ADT increased from 15 to $30 \mathrm{C}$. Solon count and frond orientation were highly correlated with the weighted difference (WDIF) between DT and NT. The weighted difference between DT and NT was equal to: (DT $x$ photoperiod) - (NT $\times$ scotoperiod). The scotoperiod was inclusive of the night interruption. Stolon count increased as the weighted NT increased relative to the weighted DT, i.e., as WDIF decreased. In contrast, frond angle relative to the soil surface, i.e., frond orientation, increased as WDIF increased. Frond unfolding rate and total plant dry weight increased as temperature increased to $\approx 25 \mathrm{C}$, then decreased.
\end{abstract}

Plant morphology is influenced by day temperature (DT), night temperature (NT), the relationship between DT and NT, and/or photoperiod (Erwin et al., 1989; Karlsson et al., 1989; Went, 1953). Karlsson et al. (1989) determined that Dendranthema stem elongation increased as DT increased and NT decreased. Lilium stem elongation responses to temperature were best described by the relationship between DT and NT, where stem elongation increased as the difference between DT and NT (DIF) increased (DT - NT) (Erwin et al., 1989). Kaczperski et al. (1989) showed that Petunia internode elongation was a function of the "weighted" DIF (WDIF) between DT and NT, where stem elongation increased as WDIF increased. WDIF was calculated from WDIF $=[$ DT $\times$ photoperiod (h)] - [NT $\times$ scotoperiod (h)].

Received for publication 3 Jan. 1992. Accepted for publication 21 Sept. 1992. Minnesota Agriculture Experiment Station no. 20,403. We appreciate the assistance of Brian Kovanda, Roar Moe, Joy Hind, Mark Smith, Martin Stockton, and Wendy Cole. Plants were donated by Green Circle Growers. The Univ. of Minnesota Agriculture Experiment Station, Michigan State Univ. Agriculture Experiment Station, American Floral Endowment, and growers supportive of Michigan State Univ. research are acknowledged for their support of this project. The cost of publishing this paper was defrayed in part by the payment of page charges. Under postal regulations, this paper therefore must be hereby marked advertisement solely to indicate this fact.
DIF's influence on stem elongation is affected by photoperiod. For instance, the effect of DIF on Fuchsia stem elongation increases as photoperiod decreases (Erwin et al., 1991). Similarly, effects of DIF on Dendranthema stem elongation increased as photoperiod decreased (R.D. Berghage, unpublished data; Erwin et al., 1992).

In Lycopersicon, Ipomea, Phaseolus, and Lilium, leaf expansion is primarily affected by absolute DT and/or NT, but not DIF (Dale, 1988; Erwin et al., 1989; Went, 1953). Fuchsia leaf expansion, however, was highly correlated with DIF (Erwin et al., 1991).

Plant development rate is a function of the actual temperature rather than DIF (Alm et al., 1988; Karlsson et al., 1988, 1989). Actual temperature effects on plant development rate are often expressed in terms of average daily temperature (ADT). Plant development rate, often expressed as leaf unfolding rate, increases linearly as ADT increases within a limited temperature range (typically between 10 and 25C) (Alm et al., 1988; Karlsson et al., 1988). Temperatures for part of a DT/NT cycle above or below the linear temperature range result in lower leaf unfolding rates than would be expected based on extrapolation from a linear model.

Fonteno(1981),Poole andConover (1981a, 1981b), and Hvolslef-Eide (1991a, 1991b) have researched light intensity and temperature effects on Nephrolepis growth. Poole and Conover (198 la, $198 \mathrm{lb}$ ) studied the effects of
DT and NT on fern growth, i.e., 'plant grade' and plant height. However, their studies focused on maximum DT and minimum NT, not true average DT, NT, or ADT.

Hvolslef-Eide (1991a, 1991b) studied how temperature and light conditions under which mother plants were grown affected mother plant morphology and subsequent growth of Nephrolepis exaltata 'Bostoniensis' explants in vitro. However, she did not study the complete effects of varying DT/NT. Instead, she evaluated the effects of NT only on plants grown with a 24C DT. Under constant temperatures, Hvolslef-Eide (1991a) determined that frond length increased as temperature increased. High DT/low NT were also reported to increase frond length. Leaflet count per frond was not determined.

We studied how Nephrolepis grown under defined environmental conditions in controlled-environment greenhouses respond to DT, NT, ADT, and two photoperiods. Models of how temperature affects frond length, leaflet count per frond, frond unfolding rate (FUR), above-ground dry weight, stolon count per plant, and frond orientation are presented. The interaction between an increase in photoperiod via night interruption lighting and photoperiod impact on Nephrolepis responses to temperature variations were tested. The objectives of our research were to 1) quantify the growth response of Nephrolepis to temperature to optimize temperature regimes in commercial fern production, and 2) study the effect of photoperiod $\mathrm{x}$ thermoperiod interaction on fern vegetative morphology.
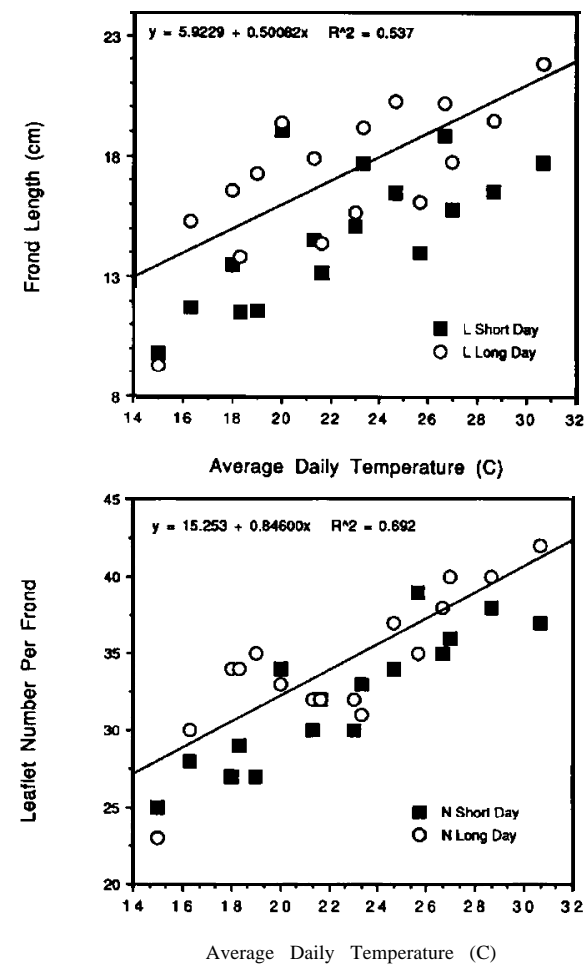

Fig. 1. Effect of average daily temperature on Nephrolepis exaltata 'Dallas Jewel' frondlength (top) and leaflet count (number) per frond (bottom). Data are presented from a representative frond taken from each plant. 
Table 1. Comparison of the $\mathrm{x}$-axis intercept and slopes of regression functions describing the effect of temperature and photoperiod on Nephrolepis exultata 'Dallas Jewel' stolon count per plant and frond orientation. Frond orientation and length was collected from a fully expanded representative frond $\approx 25$ fronds from the plant's base. Frond orientation was determined as the angle of the representative frond relative to the soil surface. Photoperiod was $9 \mathrm{~h}$ [short day (SD)] or $9 \mathrm{~h}$ plus a 2-h night interruption [long day (LD)] from incandescent lamps at a $2 \mu \mathrm{mol} \cdot \mathrm{s}^{-1} \cdot \mathrm{m}^{-2}$ irradiance. Parameters are defined as in the linear function: dependent variable $=\left(b_{0}\right)+\left(b_{1} \times X\right)$, where $X=$ the difference between day/night temperature (DIF), $b_{n}=x$-axis intercept, and $b$, = slope. Frond unfolding rate was determined by dividing total number of fronds unfolded since the experiment began by the number of days plants were treated. Dry weight was determined only on the above-ground portion of the plant. Parameters are defined as in the linear function: dependent variable $=\left(b_{0}\right)+\left(b_{1} \times X\right)+\left(b_{2} \times X^{2}\right)$ where $X=$ temperature. Significance of $a$ temperature $\times$ photoperiod interaction was determined using Snedecor and Cochran's (1967) comparison of slopes technique.

\begin{tabular}{lccccc}
\hline \hline Characteristic & Parameter & SD & LD & SD + LD & $\begin{array}{c}\text { Signif- } \\
\text { icance }\end{array}$ \\
\hline Stolon count & $\mathbf{b}_{0}$ & $0.20 \pm 1.69$ & $-0.51 \pm 1.17$ & $-0.16 \pm 1.05$ & NS \\
& $\mathbf{b}_{1}$ & $-0.05 \pm 0.01$ & $-0.04 \pm 0.01$ & $-0.04 \pm 0.01$ & NS \\
Frond orientation & $r^{2}$ & 0.62 & 0.68 & 0.61 & \\
& $\mathbf{b}_{0}$ & $59.2 \pm 4.00$ & $60.8 \pm 6.40$ & $59.7 \pm 3.90$ & Ns \\
& $\mathbf{b}_{1}$ & $0.18 \pm 0.02$ & $0.24 \pm 0.04$ & $0.21 \pm 0.02$ & Ns \\
Frond unfolding & $r^{2}$ & 0.85 & 0.75 & 0.76 & \\
& $\mathbf{b}_{0}$ & $-1.05 \pm 0.25$ & $-1.77 \pm 1.08$ & $-1.41 \pm 0.47$ & Ns \\
& $\mathbf{b}_{1}$ & $0.11 \pm 0.02$ & $0.17 \pm 0.10$ & $0.14 \pm 0.04$ & Ns \\
Plant dry weight & $\mathbf{b}_{2}$ & $-0.002 \pm 0.0005$ & $-0.003 \pm 0.002$ & $-0.003 \pm 0.0003$ & NS \\
& $r^{2}$ & 0.98 & 0.83 & 0.81 & \\
& $\mathbf{b}_{0}$ & $-4.70 \pm 5.53$ & $-13.24 \pm 5.20$ & $-8.97 \pm 3.22$ & Ns \\
& $\mathbf{b}_{1}$ & $0.53 \pm 0.51$ & $1.31 \pm 0.48$ & $0.92 \pm 0.29$ & Ns \\
& $\mathbf{b}_{2}$ & $-0.009 \pm 0.011$ & $-0.025 \pm 0.010$ & $-0.017 \pm 0.006$ & NS \\
& $r^{2}$ & 0.86 & 0.94 & 0.85 & \\
\hline
\end{tabular}

${ }^{{ }^{s} N o n s i g n i f i c a n t ~ b y ~ S n e d e c o r ~ a n d ~ C o c h r a n ' s ~(1967) ~ c o m p a r i s o n ~ o f ~ s l o p e s ~ t e c h n i q u e ~ a t ~} P=0.05$.

Nephrolepis exultata 'Dallas Jewel' plants were planted on 8 Oct. 1988 in 10.2-cm plastic pots containing a medium of equal parts sphagnum peat, perlite, and vermiculite. Plants grew for 2 weeks in a glasshouse maintained at $20 \pm$ 2C. Subsequently, 128 plants were selected for uniformity based on frond count and were moved to glasshouses with $15,20,25$, and 30 $\pm 1.5 \mathrm{C}$ setpoints. Light levels were those for natural daylight from Oct. to Dec. 1988 in East Lansing, Mich. Half of the plants (16) within each glasshouse received long days (LD) that consisted of night interruption lighting from 2200 to $0200 \mathrm{HR}$ using incandescent lamps at 2 $\mu \mathrm{mol} \cdot \mathrm{s}^{-1} \cdot \mathrm{m}^{-2}$.

Plants were moved among glasshouses at 0800 and $1700 \mathrm{HR}$ each day to yield a total of 16DT/NT combinations within each photoperiod treatment. Each treatment combination consisted of four plants. Moving plants required $15 \mathrm{~min}$. Plants held at constant temperatures were moved within the same greenhouse to account for potential thigmotropic effects resulting from moving plants daily. An opaque curtain was pulled over the plants after they were moved at $1700 \mathrm{HR}$ and was retracted before $0800 \mathrm{HR}$ to provide a 9 -h photoperiod paralleling the DT treatment. Light pollution between LD and short-day (SD) treatments within a glasshouse was eliminated by pulling an opaque curtain between plants at $1700 \mathrm{HR}$ and retracting it at $0800 \mathrm{HR}$. Fans and sensors below blankets ensured temperature control at plant level.

Plants were watered as needed to ensure that the medium was always moist for each DT/NT regime. Calcium and potassium nitrate supplied $14.3 \mathrm{~mm} \mathrm{~N}$ and $5.1 \mathrm{~mm} \mathrm{~K}$ at every watering. Electrical conductivity was maintained at 0.75 to 1 .

Data on frond count, frond length, leaflet count per frond, stolon count, frond orientation, and "above-ground" plant dry weight were collected for each plant after 92 days. Frond length, leaflet count, and frond orientation were determined from a representative fully expanded frond on each plant $\approx 25$ fronds from the plant's base to ensure valid comparisons among treatments.

Frond orientation--the representative frond angle relative to the soil surface-was measured as the angle of a fully expanded representative frond $\approx 25$ fronds from the plant's base from a line parallel to the medium surface. Therefore, plants with fronds that extended over the sides of the pot and that were lower than the soil surface had a negative frondorientation. Upright-oriented fronds had a positive frond orientation.

Data were analyzed statistically by multilinear regression analysis and analysis of variance using the SAS General Linear Models Procedure (SAS Institute, 1985). Parameter selection in regression analysis was determined by comparing $r^{2}$ and Mallow's $\mathrm{C}_{\mathrm{p}}$ of independent variables, visually inspecting the regression fit, and considering the significance of parameters within the analysis of variance procedure. Since each environmental treatment constituted a single replicate, Snedecor and Cochran's (1967) method was used to determine whether interactions were present between temperature and photoperiod.

Morphology. Frond length and leaflet count (number) per frond were highly correlated with ADT (Fig. 1, top and bottom). Linear contrasts indicated that the linear terms for both frond length and leaflet count per frond were significant at $\mathrm{P}<0.001$. Quadratic and cubic terms were not significant for either morphological characteristic $(\mathrm{a}=0.05)$. Frond length and leaflet count per frond increased linearly as ADT increased from 15 to $30 \mathrm{C}$, regardless of photoperiod (Fig. 1, top and bottom). Frond length increased from 9.3 to $21.9 \mathrm{~cm}$ and leaflet count from 23 to 42 leaflets
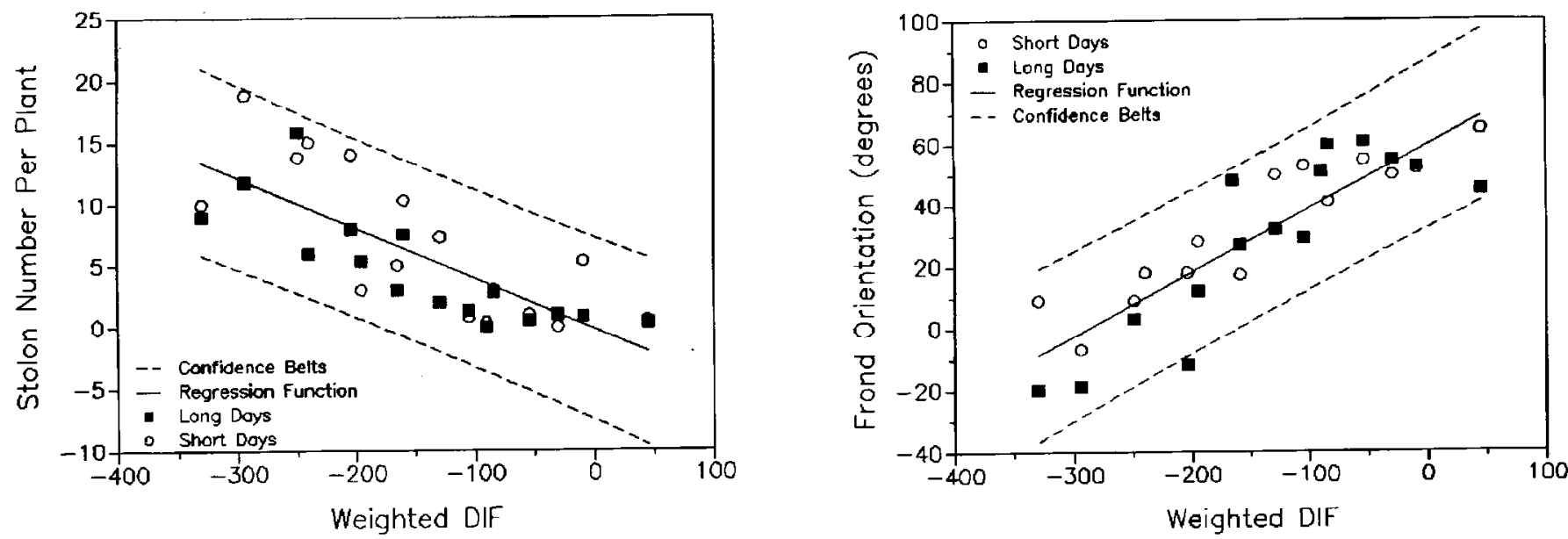

Fig. 2. Effect of weighted difference between day/night temperatures (WDIF) [degree hours $\left({ }^{\circ} \mathrm{C}\right)$ ] on (left) the number of stolons, $\geq 1 \mathrm{~cm}$, per pot and (right) frond orientation of Nephrolepis exaltata 'Dallas Jewel' after 92 days. Frond orientation $=$ angle of a representative frond relative to the soil surface. Dashed lines represent the 95\% confidence belts about the regression lines. Regression functions are shown in Table 1. 

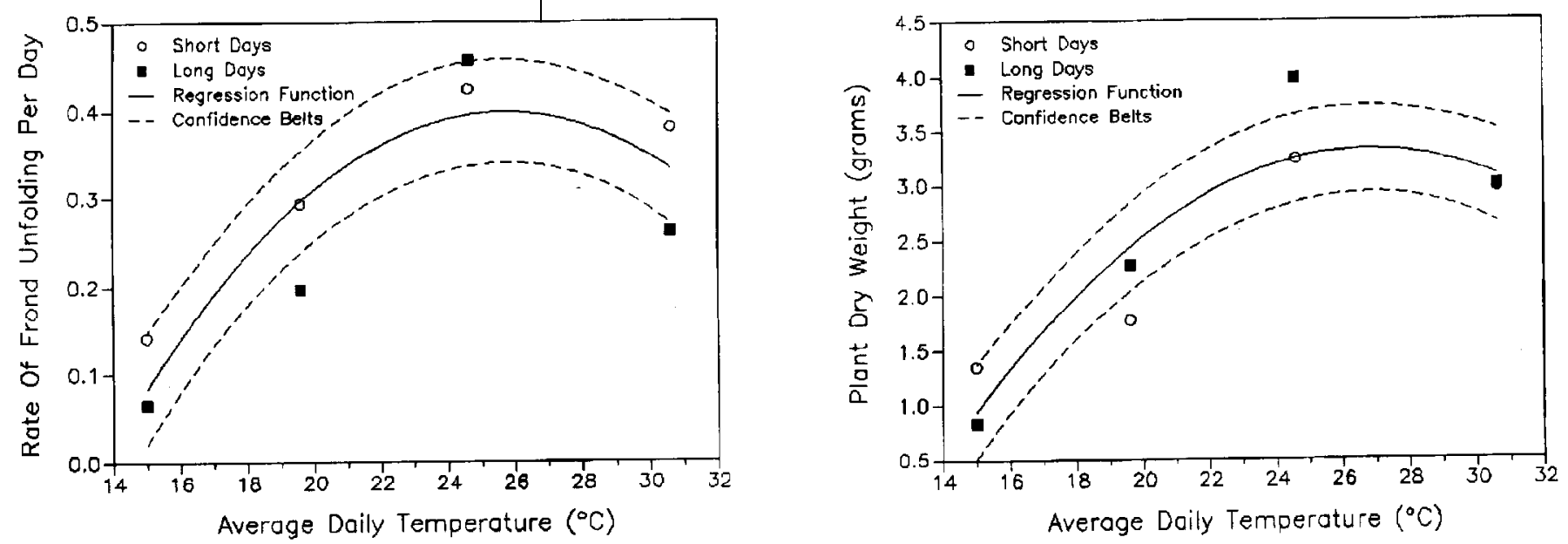

Fig. 3. Effect of temperature on Nephrolepis exaltata 'Dallas Jewel' (left) frond unfolding rate and (right) total plant dry weight after 92 days. Only treatments with constant day and night temperatures were used to calculate regression functions. Dashed lines represent the $95 \%$ confidence belts about the regression lines. Regression functions are shown in Table 1.

per frond as ADT increased from 15 to $30 \mathrm{C}$ (Fig. 1, top and bottom). Photoperiod had no significant effect on either criterion (Table 1).

Temperature effects on stolon count and frond orientation were best described by the relationship between DT and NT (DIF) rather than actual DT and NT between 15 and 30C. However, stolon count and frond orientation were more highly correlated with $\operatorname{WDIF}\left(r^{2}=\right.$ $0.72)$ than with DIF $\left(r^{2}=0.52\right)$. Linear contrasts indicated that linear terms for stolon count and frond orientation were significant at $\mathrm{P}=0.001$. Quadratic and cubic terms were not significant for either morphological characteristic. Stolon count decreased from 10.0 to 0.3 per plant as WDIF increased from -300 to $+50 \mathrm{C} / \mathrm{h}$ (Fig. 2, left). Similarly, frond orientation increased from $-20^{\circ}$ to $60^{\circ}$ as WDIF increased from -300 to $+50 \mathrm{C} / \mathrm{h}$ (Fig. 2, right). Photoperiod had no significant effect on either stolon count or frond orientation (Table 1).

Frond unfolding rate. FUR increased as DT or NT increased from 15 to $25 \mathrm{C}$ (Fig. 3, left). Linear and quadratic contrasts were significant at $P=0.001$ for this characteristic; cubic terms were not. FUR decreased if either DT or NT increased from 25 to 30C (Fig. 3, left). There was no correlation between WDIF or DIF and FUR. Photoperiod had no significant effect on FUR (Table 1).

Plant dry weight. Total above-ground plant dry weight increased as DT or NT increased from 15 to $25 \mathrm{C}$ (Fig. 3, right). Linear and quadratic contrasts were significant at $P=0.001$ for this characteristic; cubic terms were not. There was no correlation between WDIF or DIF and above-ground plant dry weight. Dry weight decreased as DT or NT increased from 25 to $30 \mathrm{C}$ (Fig. 3, right). Photoperiod had no significant effect on above-ground plant dry weight (Table 1).

The optimum temperature range for Nephrolepis, based on FUR and dry-weight gain, is much narrower than we thought. It appears to be between 23 to $27 \mathrm{C}$ for $N$. exaltata 'Dallas Jewel', as defined by FUR data in this experiment. Poole and Conover (1981a, 1981b, 1982) showed that Nephrolepis frond length and overall plant quality decreased at NT $<20$ to $15 \mathrm{C}$ or at DT $>38$ to $44 \mathrm{C}$. The differences in the optimal temperature ranges between this experiment and those of Poole and Conover (1981a, 1981b, 1982) may be due in part to variations in response to temperature that are cultivar dependent. However, in their experiments (1981a, 1981b), only maximum DT and minimum NT were evaluated and not actual average daily DT, NT, or ADT. Therefore, determining optimal growing temperatures from their data may be difficult.

Often, an increase in frond length is commercially desirable. Frond length can be maximized if DT and NT are increased to at least 30C. An optimal temperature for increasing frond length was not determined in this research, since frond length and leaflet count per frond increased with temperatures up to and including 30C, which suggests that both leaflet count and frond length had an optimal temperature $\geq 30 \mathrm{C}$. FUR and total plant dry weight decreased at temperatures $>25 \mathrm{C}$. A commercial grower must therefore make a decision as to what is more important for the market-longer fronds or more fronds-and consider the energy costs associated with heating and cooling a glasshouse.

\section{Literature Cited}

Alm, D.M., D.R. Pike, J.D. Hesketh, and E.W. Stoller. 1988. Leaf area development in some crops and weed species. Biotronics 17:29-39.

Dale, J.E. 1988. The control of leaf expansion. Annu. Rev. Plant Physiol. Mol. Biol. 39:267295.

Erwin, J.E., R.D. Heins, and M.G. Karlsson. 1989. Thermomorphogenesis in Lilium longiflorum
Thunb. 'Nellie White'. Amer. J. Bot. 76(1):47-52. Erwin, J.E., R.D. Heins, and R. Moe. 1991. Temperature and photoperiod effects on Fuchsia $\times$ hybrida. J. Amer. Soc. Hort. Sci. 116:955960.

Fonteno, W.C. 1981. Boston fern production. N.C. Flower Grower Bul. 25:3-6.

Hvoslef-Eide, A.K. 1991a. The effect of temperature, daylength and irradiance on the growth of mother plants of Nephrolepis exaltata (L.) Schott and on the subsequent growth in vitro of runner tip explants. Scientia Hort. 47: 137-147.

Hvoslef-Eide, A.K. 1991b. Mother plant temperature effects on growth of in vitro propagated daughter plants of Nephrolepis exaltata (L.) Schott. Scientia Hort. 47:149-156.

Kaczperski, M.P. 1989. Influence of temperature and irradiance on growth and development of Petunia $\times$ hybrida 'Snow Cloud'. MS Thesis, Dept. of Horticulture, Michigan State Univ., East Lansing.

Karlsson, M.G., R.D. Heins, and J.E. Erwin. 1988. Quantifying temperature-controlled leaf unfolding rates in 'Nellie White' Easter lily. J. Amer. Soc. Hort. Sci. 113:70-74

Karlsson, M.G., R.D. Heins, J.E. Erwin, and R.D. Berghage. 1989. Development rate during four phases of chrysanthemum growth as determined by preceding and prevailing temperatures. J. Amer. Soc. Hort. Sci. 114:234-240.

Poole, R.T. and C.A. Conover. 1981a. Growth response of foliage plants to night and water temperatures. HortScience 16:81-82.

Poole, R.T. and C.A. Conover. 1981b. Influence of maximum air temperatures and irrigation frequencies during high temperature periods on growth of four foliage plants. HortScience 16:556-557.

Poole, R.T. and C.A. Conover. 1982. Maximum air temperatures for summer production of foliage plants. Foliage Dig. 5(1):9-10.

SAS Institute. 1985. SAS user's guide, version 5 ed. SAS Institute, Inc., Cary, N.C.

Snedecor, G.W. and W.G. Cochran. 1967. Statistical methods. 6th ed. Iowa State Univ. Press, Ames. p. 432-436.

Went, F. 1953. The effect of temperature on plant growth. Annu. Rev. Plant Physiol. 4:347-362. 